\title{
The Source Populations Producing the Cosmic IR Background
}

\author{
Alberto Franceschini \\ Padova University, Astronomy Department, Vicolo Osservatorio 1, \\ I-35122 Padova, Italy
}

\begin{abstract}
Deep surveys performed with millimetric telescopes and with the Infrared Space Observatory have partly resolved the Cosmic IR Background (CIRB) into a population of IR sources characterized by extremely high rates of cosmological evolution (comparable to or higher than those observed for quasars). We report in this paper on early attempts to study these sources and to understand their physics. The IR multi-wavelength galaxy statistics can be explained by assuming, for the bulk of the IR population, spectra typical of starbursts, an indication that stellar more than quasar activity produces the IR emission by faint galaxies. From our fits to the observed optical-IR SEDs, the latter appear to mostly include massive galaxies hosting violent starbursts $(S F R \sim 100$ $\left.\mathrm{M}_{\odot} / \mathrm{yr}\right)$. We interpret the strong redshift-evolution as an increase with $z$ of the rate of interactions between galaxies (density evolution) and an increase of their IR luminosity due to the more abundant fuel available in the past (luminosity evolution). Our evolutionary scheme considers a bimodal star formation (SF) in galaxies, including long-lived quiescent SF, and enhanced SF taking place during transient events triggered by interactions and merging. The large energy content in the CIRB may possibly require a top-heavy stellar IMF associated with the starburst phase. The observed evolution of galaxy IR emissivity is so strong from $z=0$ to $z \sim 1$ that the CIRB spectral shape imposes a fast turnover to the evolution at $z>1$ : scenarios in which a relevant fraction of stellar formation occurs at very high- $z$ (e.g., the bulk of stars in spheroids) are not supported by our analysis.
\end{abstract}

\section{Introduction}

High-redshift galaxies and the formation of stars during cosmic history are most usually investigated by observing the stellar integrated emissions in the UV/optical/near-IR. During the last decade, however, it has become increasingly evident that observations of the high- $z$ universe at longer wavelengths do not only provide complementary information, needed, e.g., for appropriate bolometric corrections to the observed optical fluxes, but in fact also reveal entirely new phenomena which are already significantly impacting our view of galaxy formation and evolution. 


\section{The Cosmic IR Background}

The discovery of the CIRB - anticipated by a model prediction by Franceschini et al. (1994), and made possible by NASA's COBE mission - was one of the most relevant achievements of observational cosmology during the last decade, bringing to the first detection ever (with remarkably small uncertainties) of the integrated emission of distant galaxies in the form of an isotropic signal in the far-IR and sub-mm (Puget et al. 1996; Hauser et al. 1998). For comparison, background radiations observed at other wavelengths contain only modest contributions by distant galaxies and are typically dominated by AGN emissions.

In spite of the presence of bright foregrounds, there are two relatively clean spectral windows in the IR where the summed foregrounds produce two minima: the near-IR cosmological window $(2-4 \mu \mathrm{m})$ and the sub-mm window $(100-500 \mu \mathrm{m})$. Redshifted photons radiated in the two most prominent galaxy emission features - the stellar photospheric peak at $\lambda \sim 1 \mu \mathrm{m}$ and the one at $\lambda \sim 100 \mu \mathrm{m}$ due to dust re-radiation - are here observable in principle.

The original tentative detection of the CIRB signal by Puget et al. (1996) on the COBE/FIRAS maps has been confirmed with independent analyses by various other groups (Fixsen et al. 1998, who find significant isotropic flux from 200 to $1000 \mu \mathrm{m}$ ), as well as by analyses of data from the DIRBE experiment in two broad-band channels at $\lambda=140$ and $240 \mu \mathrm{m}$ (Hauser et al. 1998).

Recent analyses by Dwek \& Arendt (1998) and Gorjian, Wright, \& Chary (2000) have found also tentative signals in the near-IR cosmological window at $3.5 \mu \mathrm{m}$ and in the $\mathrm{J}$ and $\mathrm{K}$ DIRBE bands, however with large uncertainties because of the very problematic evaluation of the zodiacal scattered light. To avoid overcrowding, we report in Figure 1 only the most recent results from DIRBE (Hauser et al. 1998; Lagache et al. 1999; Finkbeiner et al. 2000) and FIRAS (Fixsen et al. 1998).

No isotropic signals are significantly detected at $4 \mu \mathrm{m}<\lambda<60 \mu \mathrm{m}$, any cosmological flux being far dominated here by the the Inter-Planetary Dust (IPD) emission. In this difficult wavelength interval, the only chances for a detection of the CIRB come from high-energy observations of distant blazars (Stecker, de Jager, \& Salomon 1992). The idea is to infer the CIRB intensity from combined X-ray and $\mathrm{GeV}$ and $\mathrm{TeV} \gamma$-ray observations of blazars by exploiting the $\gamma-\gamma$ pair-production interaction between the source high energy photons and photons of the CIRB. The absorption cross-section of $\gamma$-rays of energy $E_{\gamma}(\mathrm{TeV})$ has a maximum for IR photons with energies obeying the condition $\epsilon_{\text {max }}=2\left(\mathrm{~m}_{\mathrm{e}} \mathrm{c}^{2}\right)^{2} / E_{\gamma}$, or equivalently $\lambda_{\text {peak }}(\mu \mathrm{m}) \simeq(1.24 \pm 0.6) E_{\gamma}(\mathrm{TeV})$ (Stecker et al. 1992).

The optical depth for a high-energy photon $E_{\gamma}$ traveling through a cosmic medium filled with low-energy photons with density $\rho(z)$ from $z_{e}$ to the present time can be approximated as (Coppi \& Aharonian 1999):

$$
\tau_{\gamma \gamma}\left(E_{0}, z_{e}\right) \simeq 0.063\left(\frac{E_{\gamma}}{\mathrm{TeV}}\right)\left(\frac{\nu I_{\nu}}{\mathrm{nW} / \mathrm{m}^{2} / \mathrm{sr}}\right)\left(\frac{z_{e}}{0.1}\right) h_{60}^{-1} .
$$

Interesting applications of this concept were made possible when data from the Compton Gamma Ray Observatory and from hard X-ray space telescopes were combined with $\gamma$-ray observations at $\mathrm{TeV}$ energies by the Whipple, HEGRA 
and other Cherenkov observatories on Earth. Stanev \& Franceschini (1998) in particular have discussed model-independent upper limits on the CIRB (no $a$ priori guess on the CIRB spectrum), using HEGRA data for the blazar Mkn 501 $(z=0.034)$ during an outburst in 1997, under the assumption that the highenergy source spectrum is the flattest allowed by the data. These limits (dotted histogram in Fig. 1) get quite close to the CIRB background already resolved by the ISO mid-IR deep surveys (see Sect. 4 below).

Krawczynski et al. (1999) have combined the observations of Mkn 501 during the 1997 outburst with simultaneous X-ray data from Rossi XTE and BeppoSAX, providing a high-quality description of the whole high-energy spectrum. These data are very well fitted by a Synchrotron Self Compton (SSC) model in which the spectrum at $\nu=10^{27} \mathrm{~Hz}$ is produced by Inverse Compton off the hard $\mathrm{X}$-ray spectrum at $\nu=10^{18} \mathrm{~Hz}$. This model is used to derive $\tau_{\gamma \gamma}$ as a function of energy and a constraint on the spectral intensity of the CIRB. The result is compatible with the limits by Stanev \& Franceschini (1998) and permits a tentative estimate of the CIRB intensity in the interval from $\lambda=20$ to $40 \mu \mathrm{m}$ (see dotted line in Fig. 1).

A less model dependent constraint is set by the observations of purely powerlaw blazar spectra around $E_{\gamma} \simeq 1 \mathrm{TeV}$, which translates into the upper limits around $10 \mathrm{nW} / \mathrm{m}^{2} / \mathrm{sr}$ at $\lambda \simeq 1 \mu \mathrm{m}$ as shown in Fig. 1 . Substantially exceeding this limit, as suggested by some authors, would imply either very ad hoc $\gamma$-ray source spectra or new physics (Harwit, Protheroe, \& Biermann 1999).

Altogether, there is now ample consensus even on details of the CIRB spectral intensity, at least from 140 to $500 \mu \mathrm{m}$ where it is most reliably measured. The CIRB flux has in particular stabilized at values $\nu I_{\nu} \simeq 20$ and $\nu I_{\nu} \simeq 15 \mathrm{nW} / \mathrm{m}^{2} / \mathrm{sr}$ at $\lambda=140$ and $240 \mu \mathrm{m}$.

The measurement of the CIRB provides the global energy density radiated by cosmic sources at any redshift. Two concomitant facts - the very strong Kcorrection for galaxies in the far-IR/sub-mm due to the very steep and featureless dust spectra, and their relative robustness due to the modest dependence of dust equilibrium temperature $T$ on the radiation field intensity - have suggested the use of the CIRB spectrum to infer the evolution of the long-wavelength galaxy emissivity as a function of redshift (Gispert, Lagache, \& Puget 2000). Indeed, while the peak intensity at $\lambda=100$ to $200 \mu \mathrm{m}$ constrains the integrated galaxy emissivity in the redshift interval from $z=0$ to $z=1$, the low foreground contamination at $\lambda>200 \mu \mathrm{m}$ permits setting important constraints on it at $z>1$.

Between 100 and $1000 \mu \mathrm{m}$ the integrated CIRB intensity turns out to be $\sim(30 \pm 5) 10^{-9} \mathrm{~W} / \mathrm{m}^{2} / \mathrm{sr}$. In addition to this measured part of the CIRB, one has to consider the presently unmeasurable fraction resident in the wavelength range between 100 and $10 \mu \mathrm{m}$. Adopting model extrapolations as in Fig. 1 consistent with the constraints set by the cosmic opacity observations, the total energy density between 7 and $1000 \mu \mathrm{m}$ amounts to

$$
\left.\nu I(\nu)\right|_{F I R} \simeq 4010^{-9} \mathrm{~W} / \mathrm{m}^{2} / \mathrm{sr}
$$

This flux compares with the integrated bolometric emission by distant galaxies between 0.1 and $7 \mu \mathrm{m}$ (the "optical background"), for which we adopt 


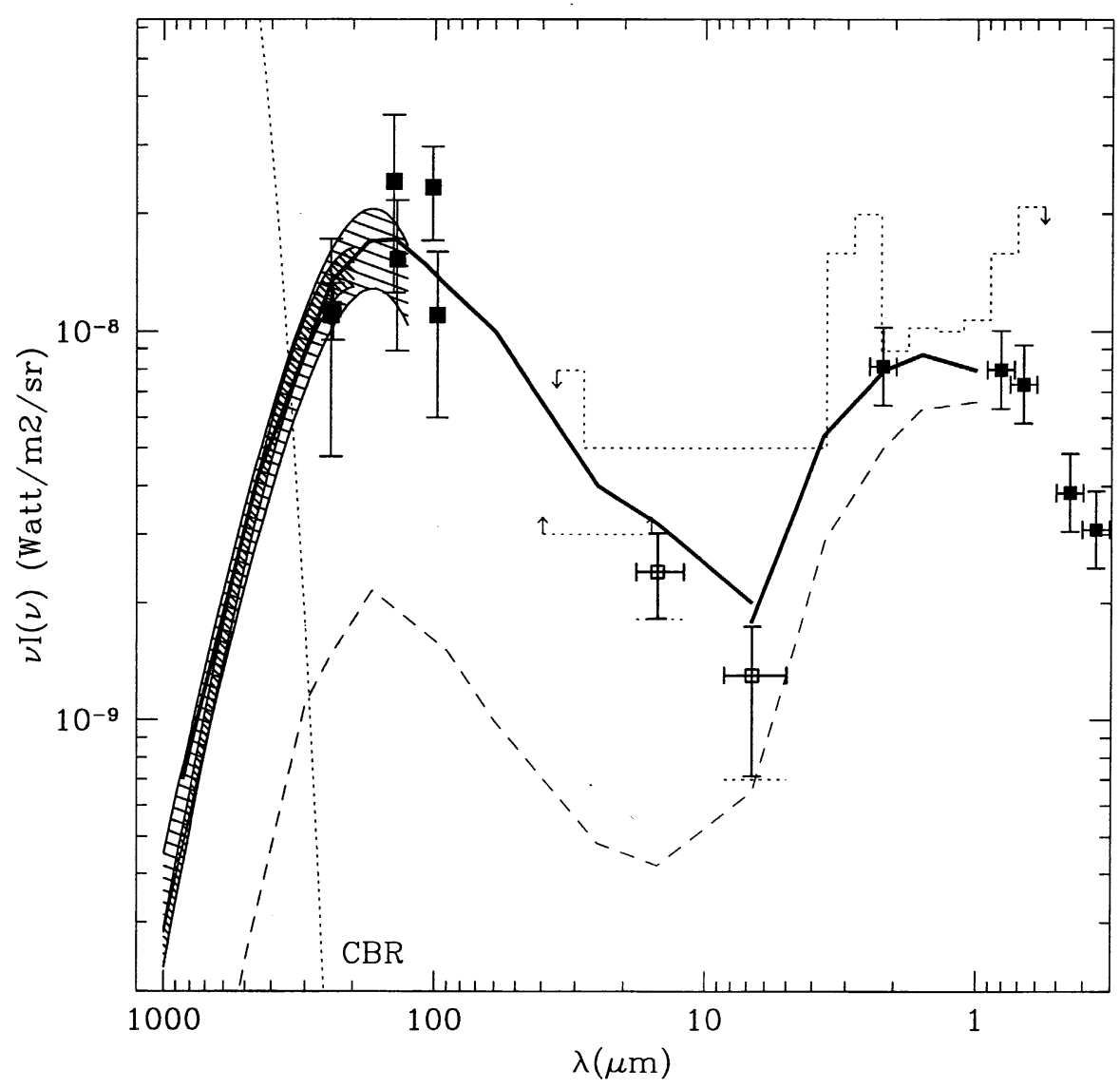

Figure 1. The Cosmic Infrared Background (CIRB) spectrum as measured by independent groups in the all-sky COBE maps (e.g., Hauser et al. 1998; Lagache et al. 1999), compared with estimates of the optical background based on ultradeep surveys (Madau \& Pozzetti 2000). The two mid-IR data points are the resolved fraction of the CIRB by the deep ISO surveys IGTES, while the dotted histogram are limits set by $\mathrm{TeV} \gamma$-ray cosmic opacity. The lower dashed line marks the expectation based on the assumption that the IR emissivity of galaxies does not change with cosmic time. The thick line is the predicted CIRB intensity by the presently discussed model for IR galaxy evolution. 
the value given by Madau \& Pozzetti (2000):

$$
\left.\nu I(\nu)\right|_{\text {opt }} \simeq(17 \pm 3) 10^{-9} \mathrm{~W} / \mathrm{m}^{2} / \mathrm{sr} .
$$

This latter has been obtained by counting all galaxies detected between 0.3 and $3 \mu \mathrm{m}$ by HST down to the faintest detectable limits, by exploiting the fact that optical counts show a clear convergence at magnitudes $m_{A B} \geq 22$.

As we see, the directly measured part of the CIRB already sets a relevant constraint on the evolution of cosmic sources, if we consider that for local galaxies on average only $30 \%$ of the bolometric flux is absorbed by dust and re-emitted in the far-IR. The fact that the CIRB intensity exceeds the optical background suggests that galaxies in the past should have been much more "active" in the far-IR than in the optical, and very luminous in an absolute sense. A substantial fraction of the whole energy emitted by high-redshift galaxies should have been reprocessed by dust at long wavelengths.

\section{Resolving the CIRB into Faint Discrete Sources}

\subsection{Deep Sky Surveys with the Infrared Space Observatory (ISO)}

The ISO observatory (a 60 -cm cryogenic space telescope operated by ESA between 1995 and 1998) included two instruments of cosmological interest: a midIR camera (ISOCAM, 4 to $18 \mu \mathrm{m}$ ) and a far-IR imaging photometer (ISOPHOT, 60 to $200 \mu \mathrm{m}$ ). The vast improvement in sensitivity offered by ISO with respect to IRAS justified spending a substantial amount of observing time on deep surveys at mid- and far-IR wavelengths, also motivated by the concomitant discovery of the CIRB. We defer to Elbaz et al. (1999) and Franceschini et al. (2000) for more details on the main ISO survey programs; we simply recall here a few basic facts.

Five extragalactic surveys with the LW2 and LW3 filters have been performed in the ISOCAM Guaranteed Time Surveys (IGTES). A total area of 1.5 square degrees have been surveyed and more than one thousand sources have been detected (Elbaz et al. 1999).

The European Large Area ISO Survey (ELAIS) was the largest program in the ISO Open Time (Oliver et al. 2000). A total of 12 square degrees have been surveyed at $15 \mu \mathrm{m}$ with ISOCAM and at $90 \mu \mathrm{m}$ with ISOPHOT. ELAIS was split into 4 fields of comparable size, 3 in the North and one in the South.

The two Hubble Deep Field areas (North and South), including the Flanking Fields for a total of $\sim 50$ sq. arcmin, have been deeply surveyed by ISOCAM at 6.7 and $15 \mu \mathrm{m}$, achieving completeness to a limiting flux of $100 \mu \mathrm{Jy}$ at $15 \mu \mathrm{m}$. In the inner 5 square arcmin, the exceptional images of HST provided detailed morphological information for ISO galaxies at any redshift. Thanks to the variety of photometric data and the almost complete redshift information available (Rowan-Robinson et al. 1997; Aussel et al. 1999), these surveys are providing the most detailed characterization of the faint IR source population. The redshift distributions show an excess number of sources between $z=0.5$ and $z=1$.

FIRBACK is a set of deep cosmological surveys carried out with ISOPHOT, specifically aimed at detecting at $170 \mu \mathrm{m}$ the sources of the CIRB (Puget et al. 1999). This survey is limited by extragalactic confusion in the large 

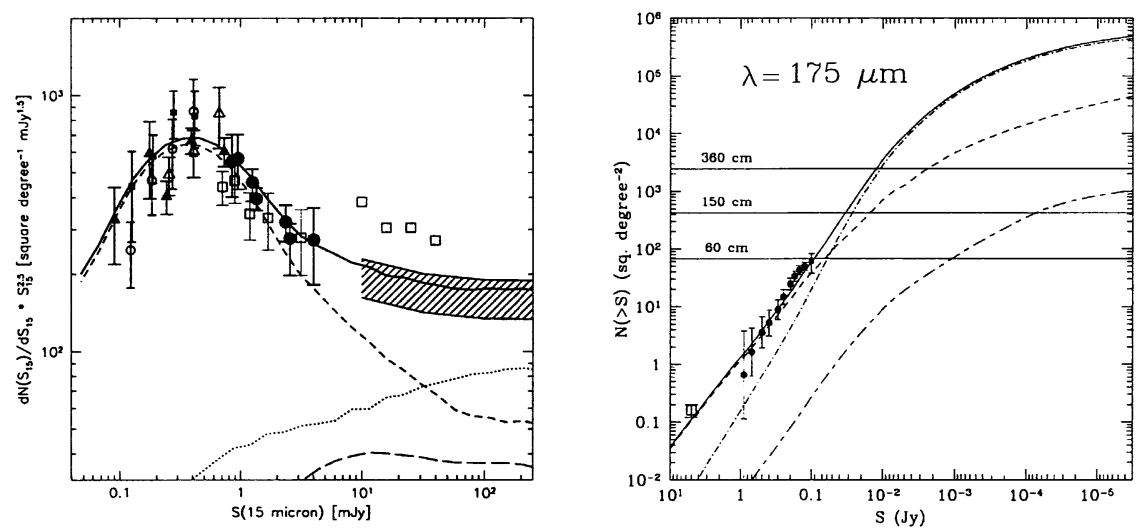

Figure 2. Left panel: normalized differential counts at $\lambda_{\text {eff }}=15 \mu \mathrm{m}$ (Elbaz et al. 1999). Right panel: integral counts at $175 \mu \mathrm{m}$. See text for the meaning of the lines. The horizontal lines mark the limit of confusion for various telescope sizes.

ISOPHOT beam $(90 \mathrm{arcsec})$ to $S_{170} \geq 100 \mathrm{mJy}$. Roughly 300 sources were detected; these are presently targets of follow-up observations exploiting deep radio data to help reduce the ISO error box.

The most detailed information on faint IR counts comes from the ISO $15 \mu \mathrm{m}$ surveys. The differential counts based on these data and normalized to a euclidean law $S^{-2.5}$, shown in Figure 2, reveal a remarkable agreement between samples selected in seven independent sky areas. A total of 614 highly reliable sources have been used in the Figure, out of a total of 993 catalogued sources (Elbaz et al. 1999).

The combined $15 \mu \mathrm{m}$ differential counts display various remarkable features: a roughly euclidean slope from the brightest IRAS observed fluxes down to $S_{15} \sim 5 \mathrm{mJy}$; a sudden upturn at $S_{15}<3 \mathrm{mJy}$, where the counts increase as $d N \propto S^{-3.1} d S$ to $S_{15} \sim 0.4 \mathrm{mJy}$; and finally the evidence for a flattening below $S_{15} \sim 0.3 \mathrm{mJy}$ (where the slope becomes quickly sub-euclidean, $S^{-2}$ ). Note that the sudden change in slope and the convergence is supported by three independent surveys.

The areal density of ISOCAM $15 \mu \mathrm{m}$ sources is $\sim 5 \operatorname{arcmin}^{-2}$ at the survey limits. If we consider that the diffraction-limited PSF has FWHM 7 arcsec, and for a slope of the counts $\beta \sim-2$, this density is close to the ISO confusion limit at $15 \mu \mathrm{m}$ of 7 sources/arcmin ${ }^{2}$ (Franceschini 2000). Confusion will likely remain a limiting factor for NASA's SIRTF mission, in spite of the moderately larger primary collector.

Obviously, the ISO far-IR selected samples are even more seriously affected by confusion. The data points for the $175 \mu \mathrm{m}$ integral counts reported in Figure 2 come from the FIRBACK survey (Puget et al. 1999; Dole et al. 2000). These surveys have essentially attained the ISOPHOT confusion limit. 

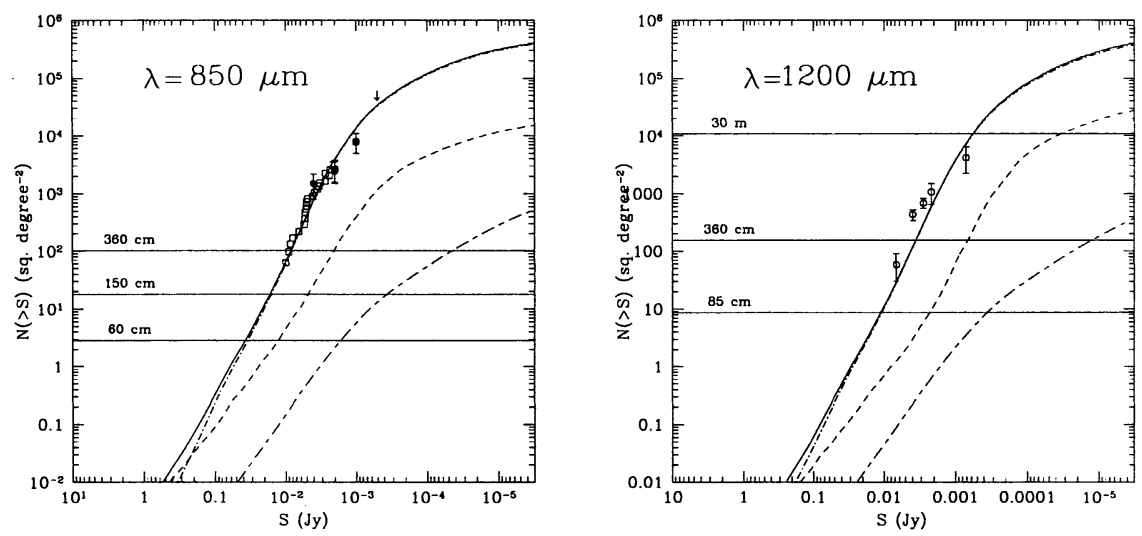

Figure 3. Integral counts at $\lambda_{\text {eff }}=850 \mu m$ (left panel) and 1200 $\mu \mathrm{m}$ (right panel), data taken from Bertoldi et al. 2000 .

\subsection{Surveys at Millimetric Wavelengths}

Surveys in the sub-millimeter offer the unique advantage of naturally generating volume-limited samples from flux-limited observations. This property is due to the peculiar shape of galaxy spectra in the sub-mm, with an extremely steep slope from $1 \mathrm{~mm}$ to $200 \mu \mathrm{m}$ [roughly $L(\nu) \propto \nu^{3.5}$ ]. A further related advantage of $\mathrm{mm}$ surveys is that local galaxies emit very modestly at these wavelengths. Altogether, a sensitive sub-mm survey will avoid local objects (stars and nearby galaxies) and will preferentially select sources at very high redshifts: a direct picture of the high-redshift universe, impossible to obtain at other frequencies.

Important discoveries have come from the implementations of arrays of bolometers (SCUBA and MAMBO) on JCMT and IRAM. SCUBA/JCMT has permitted resolving $\sim 30 \%$ of the CIRB background at $850 \mu \mathrm{m}$ into a population of faint distant, mostly high- $z$ sources. The extragalactic source counts at 850 and $1200 \mu \mathrm{m}$, reported in Figure 3, show a dramatic departure from the euclidean law $\left[d N \propto S^{-3} d S\right.$ in the crucial flux-density interval from 1 to $\left.10 \mathrm{mJy}\right]$, a clear signature of the strong evolution and high redshift of mm-selected sources.

\section{A Panchromatic View of IR Galaxy Evolution}

The deep counts at the various observed wavelengths in Figs. 2 and 3 display more or less significant departures from no-evolution behavior. The differential counts at $15 \mu \mathrm{m}$ shown in Fig. 2 are particularly well defined over a wide flux interval (including the bright IRAS counts at $12 \mu \mathrm{m}$ ). These data contain some indications about the properties of the evolving populations. The almost flat (euclidean) normalized counts extending from the bright IRAS fluxes down to a few mJy, followed by the sudden upturn below, suggest that it is possibly a locally small fraction of IR galaxies which evolve with high rates back in cosmic time. 
We have reproduced the IR counts in Fig. 2 with the contribution of two source populations, one non evolving (with local luminosity function consistent with the IRAS $12 \mu \mathrm{m} \mathrm{LLF}$ ), the other strongly evolving in cosmic time both in comoving number density and in luminosity as:

$$
\begin{gathered}
\rho(L[z], z)=\rho_{0}\left(L_{0}\right) \times(1+z)^{4.6}, \quad L(z)=L_{0} \times(1+z)^{2} \quad z<z_{\text {break }} \\
\rho(L[z], z)=\rho_{0}\left(L_{0}\right) \times\left(1+z_{\text {break }}\right)^{4.6}, L(z)=L_{0} \times\left(1+z_{\text {break }}\right)^{2} \quad z_{\text {break }}<z<z_{\text {max }}
\end{gathered}
$$

with $z_{\text {break }}=0.8$ and $z_{\max }=3$. The fit to the observed counts has a sensible dependence on the assumed values for the geometrical parameters of the universe: this solution assumes $\Omega_{m}=0.2, \Omega_{\Lambda}=0.8$ and $\mathrm{H}_{0}=50 \mathrm{~km} / \mathrm{s} / \mathrm{Mpc}$. A third source population consists of type-I AGNs, assumed to evolve in luminosity as $L(z)=L(0) \times(1+z)^{3}$ up to $z=1.5$. The local fraction of the evolving starburst population is assumed to be $\sim 10$ percent of the total, roughly consistent with the observed fraction of interacting galaxies. Clearly this evolutionary scheme is one among various possible solutions still allowed by the presently available data. Other fitting schemes have been discussed by Roche and Eales (1999) and $\mathrm{Xu}(2000)$.

Deep surveys at various IR/sub-mm wavelengths can be exploited to simultaneously constrain the evolutionary properties and broad-band spectra of faint IR sources. Franceschini et al. (2000) have fitted the IR multi-wavelength statistics with the above model by adopting suitable SEDs for IR sources. Without entering into unnecessary details (e.g., galaxy SEDs depend to some extent on the bolometric far-IR luminosity), we note here that if we adopt as representative for the evolving population the IR SED of the ultra-luminous galaxy Arp 220, the consequence would be that all far-IR counts (and the CIRB intensity, see Elbaz et al. 2000) would be exceeded by substantial factors. On the contrary, if we assume for the IR evolving sources a more typical starburst spectrum (like the one of M82, in all ways similar to those of other luminous starbursts observed by ISO), then most of the observed properties of far-IR galaxy samples can be appropriately reproduced. Best-fits to the counts based on our reference model using a spectral template similar to the M82 spectrum are given in Figs. 2 and 3.

The $15 \mu \mathrm{m}$ counts display a remarkable convergence below $S_{15} \sim 0.2 \mathrm{mJy}$ (Fig. 2). The observed asymptotic slope, flatter than -2 in differential count units, implies a modest contribution to the integrated CIRB flux by sources fainter than this limit. A meaningful estimate of the CIRB flux can then be obtained from direct integration of the observed mid-IR counts (the two data points at 15 and $7 \mu \mathrm{m}$ in Fig. 1). This implies a value of $2.6 \pm 0.5 \mathrm{nW} / \mathrm{m}^{2} / \mathrm{sr}$ at $15 \mu \mathrm{m}$ contributed by LW3 sources brighter than $S_{15}=40 \mu \mathrm{Jy}$ (Elbaz et al. 2000). Our evolutionary model implies that the expected contribution of fainter sources would bring the total background to $3.3 \mathrm{nW} / \mathrm{m}^{2} / \mathrm{sr}$. This limit is close to the upper boundary set by the observed $\mathrm{TeV} \gamma$-ray cosmic opacity (dotted histogram in Fig. 1). It appears that the ISOCAM surveys have resolved a significant (50-70\%) fraction of the CIRB intensity in the mid-IR.

The good match to the multi-wavelength counts and to the spectral intensity of the CIRB (heavy line in Fig. 1) found by assuming a typical starburst spectrum for the evolving population is already a clear indication that the faint $I R$-selected population is likely dominated by star-formation processes rather than 

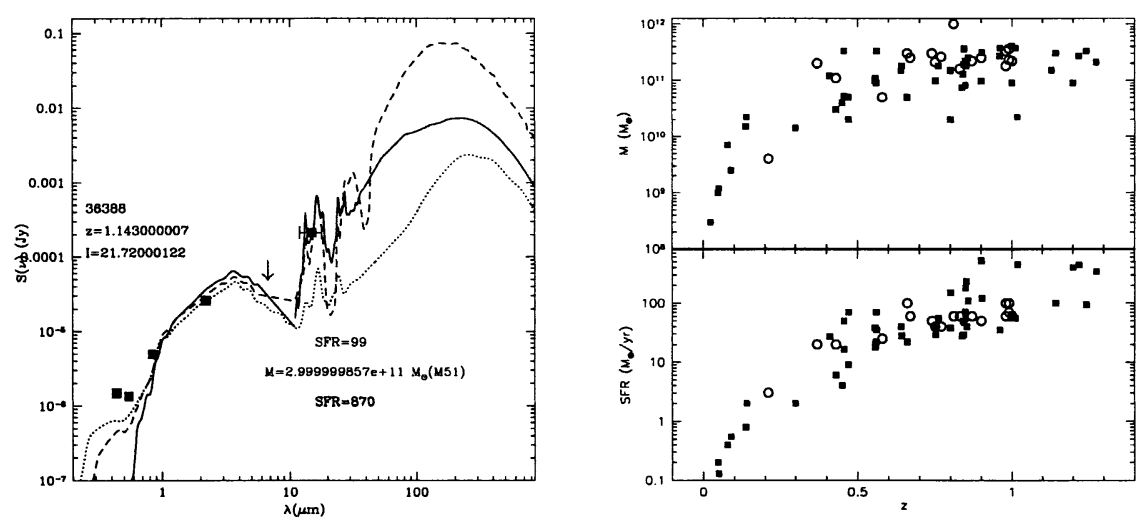

Figure 4. Left panel: broad-band spectrum of a mid-IR source selected by ISOCAM LW3 in the Hubble Deep Field North (Aussel et al. 1999), compared with the SEDs of M82 (thick continuous line), Arp 220 (dashed line), and M51 (dotted line). Estimates of the SF rate and stellar mass are indicated. Right panel: masses and starformation rates of $15 \mu \mathrm{m}$ sources detected in the HDFN and CFRS $1415+52$ fields.

AGN emission, which would produce completely different IR spectra. This also supports the conclusion that the population detected by ISO in the mid-IR not only contributes a major fraction of the CIRB at $15 \mu \mathrm{m}$, but is also responsible for a majority contribution to the CIRB at any wavelength.

\section{Hints on the Physics of the Rapidly Evolving Source Population}

Because of the very different K-corrections, faint sources selected by millimetric telescopes and by ISO display quite complementary properties in terms of redshift coverage (typically $z<1$ for sources detected by ISO and $z>1$ by SCUBA) and source luminosities $\left(L_{b o l}<10^{12} \mathrm{~L}_{\odot}\right.$ by ISO, and larger by SCUBA).

However, the deep ISO mid-IR surveys provide various advantages when investigating the nature of the source population producing the CIRB: they are responsible for a large fraction of the CIRB, their optical counterparts are easy to identify since they are at moderate redshift, and catalogues of thousands of ISO mid-IR sources are available in many independent sky areas. The situation is quite different for the high- $z$ sources selected by millimetric telescopes: their optical counterparts are so faint that they have in only a few cases been reliably identified, and in addition their volume density is so low that they can produce only a small fraction of the CIRB intensity.

The ISO surveys in the Hubble Deep Fields and the CFRS areas have been particularly well studied. The optical-IR SED of a typical faint $15 \mu \mathrm{m}$ source at $z=1.14$ is reported in Figure 4. The dotted line fitting the optical-NIR spectrum and corresponding to the SED of a quiescent spiral (M51) falls short by a factor $\sim 10$ of explaining the mid-IR emission, whereas SEDs of IR starbursts (e.g., 
Arp 220 and M82) provide more consistent fits to the global SED. The vast majority of faint ISO sources show similar mid-IR flux excesses.

The morphological information available from HST imaging data indicates that a large fraction (30 to 50\%) show evidence of peculiarities and multiple structures (Aussel et al. 1999), in keeping with the local evidence that galaxy interactions are the primary trigger of luminous IR starbursts. These sources are also typically found in galaxy groups (Cohen et al. 2000).

Although the UV rest-frame spectra do not reveal strong emission lines (Flores et al. 1999), Rigopoulou et al. (2000) have found that the $\mathrm{H}_{\alpha}$ line redshifted into the near-IR is strong $(\mathrm{EW}>50 \AA)$, indicating substantial rates of SF after de-reddening corrections, and demonstrating that these optically faint but IR luminous sources are indeed powered by an ongoing massive dusty starburst.

We have estimated the baryonic mass in these galaxies from fits of template SEDs of local galaxies to the observed near-IR broad-band spectrum (assuming a Salpeter IMF between 0.15 to $100 \mathrm{M}_{\odot}$ ). Our estimated values of the baryonic masses $\left(\sim 10^{11} \mathrm{M}_{\odot}\right.$ at $z>0.4$, with 1 dex typical spread, see Figure 4$)$ indicate that already evolved and massive galaxies preferentially host the powerful starbursts.

To evaluate the other fundamental physical parameter - the star-formation rate (SFR) - we have exploited the mid-IR flux as an alternative to the (heavily extinguished) optical-UV emissions. The capability of the mid-IR flux to measure the SFR is discussed by Vigroux et al. (1998): this flux appears tightly correlated with the bolometric (mostly far-IR) emission, which is the most robust measure of the number of massive reddened newly-formed stars.

The rates of SF indicated by the fits to the mid-IR flux for sources at $z>0.5$ range from several tens to a few hundred solar masses/yr, i.e., a substantial factor higher than found for optically-selected galaxies. The extinction corrected $H_{\alpha}$ flux turns out to explain only 20-30\% of the bolometric far-IR emission: the bulk of SF in luminous IR galaxies is unobservable in the optical.

Altogether, the galaxy population dominating the faint mid-IR counts and significantly contributing to the bolometric CIRB intensity appears to be composed of luminous $\left(L_{b o l} \sim 10^{11}-10^{12} \mathrm{~L}_{\odot}\right)$ starbursts in massive $\left(M \sim 10^{11} \mathrm{M}_{\odot}\right)$ galaxies at $z \sim 0.5-1$, observed during a phase of intense star formation $\left(S F R \sim 100 \mathrm{M}_{\odot} / \mathrm{yr}\right.$ ). The typically red colors of these systems suggest that they are mostly unrelated to the faint blue galaxy population dominating the optical counts (Ellis 1997), and should be considered as an independent manifestation of (optically hidden) star formation (Aussel 1998).

As suggested by several authors, the similarity in properties (bolometric luminosities, SEDs) between this high- $z$ population and local ultra-luminous IR galaxies argues in favour of the idea that these represent the long-sought "primeval galaxies", those in particular producing the local massive elliptical and S0 galaxies. This is also supported by estimates of the volume density of these objects in the field $\sim 2-4 \times 10^{-4} \mathrm{Mpc}^{-3}$, high enough to allow most of the E/S0 to be formed in this way (Lilly et al. 1999).

By continuity, the less extreme starbursts $\left(L \sim 10^{11}-10^{12} \mathrm{~L}_{\odot}\right)$ discovered by ISOCAM at lower redshifts may be related to the origin of lower mass spheroids and spheroidal components in later morphological type galaxies. 


\subsection{AGN Contributions to Source Energetics}

How much of the bolometric flux in these IR/mm sources could be contributed by gravitational accretion rather than by stellar emission? Answering this question is difficult because the optical-UV-soft X-ray primary source spectrum in highredshift IR-mm sources is almost completely re-processed by dust into an IR spectrum insensitive to the properties of the primary incident flux.

The CHANDRA X-ray observatory has permitted probing very deeply into the nature of the high- $z$ SCUBA population, using the hard X-ray flux as a diagnostic tool (starbursts are weaker X-ray emitters than any kind of AGNs). Several hard X-ray and $850 \mu \mathrm{m}$ source samples detected in various sky areas have been cross-correlated by Fabian et al. (2000), Hornschemeier et al. (2000), and Barger et al. (2000), but only a very small percentage of the sources are in common, the two classes being essentially orthogonal. Unless all these are Compton-thick and any hard X-ray scattered photons are also photo-electrically absorbed, the conclusion is that the bulk of the emission by high-luminosity SCUBA sources is due to star formation (this does not exclude that minor AGN contributions are present in the active IR population. Risaliti et al. 2000 find trace AGN emission in $60-70 \%$ of local ULIRGs based on BeppoSAX hard $\mathrm{X}$-ray data).

While the detailed interplay between starburst and AGN is still an open issue, the estimated fraction of the CIRB at $850 \mu \mathrm{m}$ due to AGNs is not likely larger than $10 \%$ (Barger et al. 2000). Preliminary results of spectroscopic studies of faint ISO mid-IR sources by Rigopoulou et al. (2000) show the $H \alpha$ line profiles and the 15 to $7 \mu \mathrm{m}$ flux ratios to be consistent with the large majority being powered by a SB rather than an AGN, hence confirming an overall AGN contribution to the bolometric CIRB likely around $10 \%$.

\section{Global Properties: the Comoving SFR and Constraints from Back- ground Observations}

Unfortunately, an independent assessment of the evolutionary SFR density is not possible until complete samples of faint IR sources with identifications become available. At present, only model-dependent estimates of the SFR density as a function of redshift are possible. The prediction based on our presently discussed evolution scheme is reported in Figure 5. There is a clear indication here that the contribution of IR-selected sources to the luminosity density at high- $z$ should significantly exceed those based on optically selected sources, and that the excess may be progressive with redshift up to $z \sim 1$. Consider in any case that the optical and IR estimates of the SFR in Fig. 5 refer to largely distinct source populations (extinction-corrected optical fluxes account on average for only 20$30 \%$ of the far-IR emission, see Sect. 5). The global value of the SFR can then be obtained by adding the separate optical and IR contributions.

Fig. 5 illustrates that the estimated rate of evolution for the IR volume emissivity of galaxies at $z<1$ (top line) is even higher than that of optical/Xray AGNs (bottom line). This evolution inferred from the IR observations should however level off at higher $z$, to allow consistency with the observed $z$-distributions for faint ISOCAM sources. A further important constraint in this sense comes from the observed shape of the CIRB and its apparent fast 


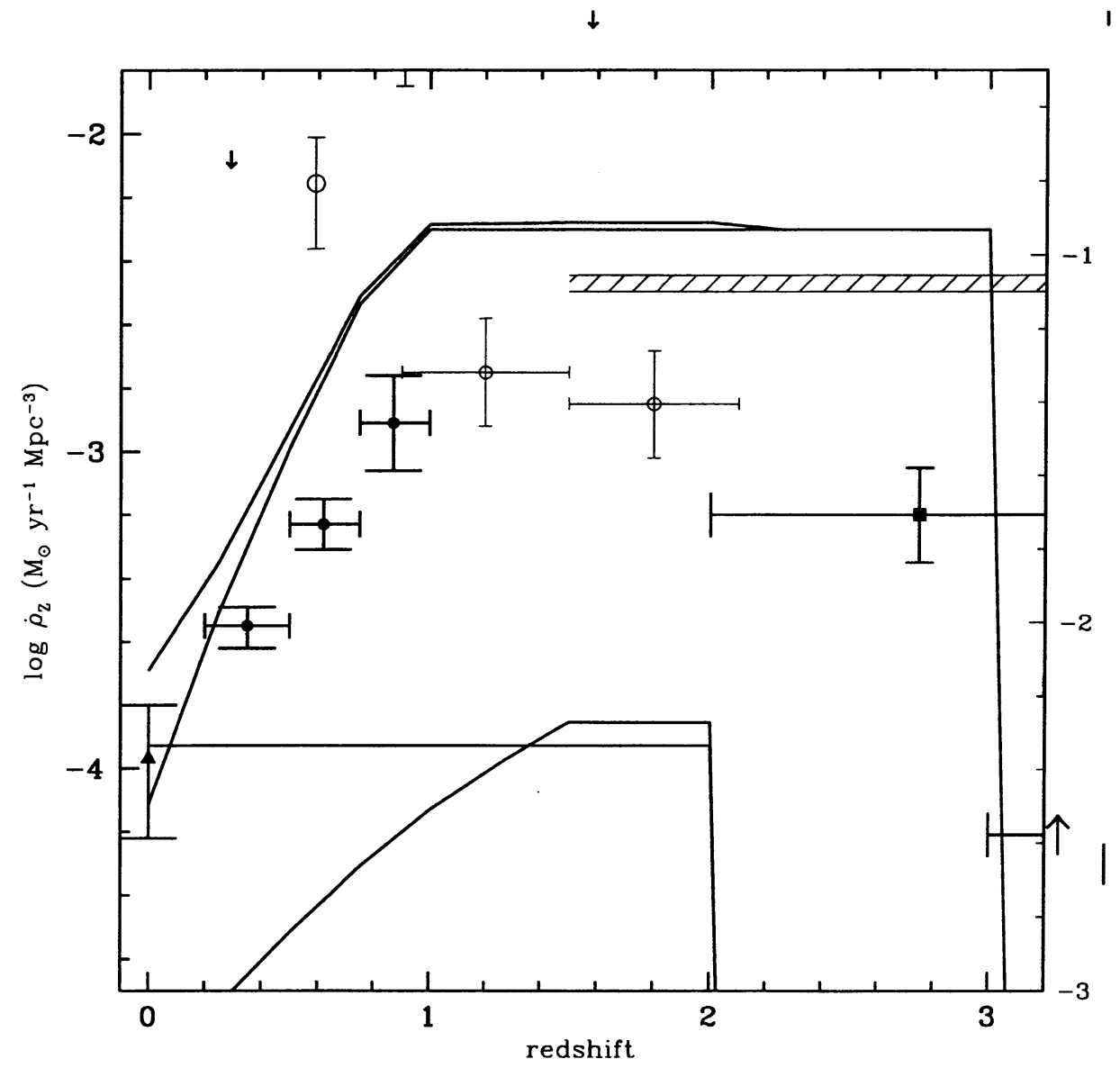

Figure 5. Evolution of the metal production rates (left axis) and of the star formation rates (in $\mathrm{M}_{\odot} / \mathrm{yr}$, right axis) based on the models of IR counts and $z$-distributions in Sect 4. Data points come from optical observations. From top to bottom, the lines are: total, starburst, quiescent population, and AGNs. The shaded horizontal line is an evaluation of the average SFR in spheroidal galaxies by Mushotzky \& Loewenstein (1997). 
turnover at $\lambda>200 \mu \mathrm{m}$ : fitting it with standard dust-emission spectra implies a maximum in the comoving galaxy IR emissivity close to $z=1$ (see also Gispert et al. 2000).

Altogether these results indicate that the history of galaxy long-wavelength emissivity does not probably follow a path very dissimilar from that revealed by optical-UV observations, showing a similar peak activity around $z \sim 1-1.5$, rather than being confined to the very high-redshifts as sometimes has been suggested. This confirms that the bulk of the galaxy activity is to be placed around $z=1$, which is evident from Fig. 5 if the dependence of the cosmological timescale on redshift is considered (e.g., Harwit 1998).

\subsection{Energy Constraints from Background Observations}

Further constraints on the high-redshift far-IR/sub-mm population can be inferred from observations of the global energetics residing in the CIRB and optical backgrounds.

Let us assume that a fraction $f_{*}$ of the universal mass density in baryons $\rho_{b}$ undergoes at redshift $z_{*}$ a transformation (either processed in stars or by gravitational fields) with radiative efficiency $\epsilon$. The locally observed energy density of the remnant photons is (e.g., Burigana et al. 1997; Harwit 1998):

$$
\rho_{\gamma}=\rho_{b} \frac{c^{2} \epsilon f_{*}}{\left(1+z_{*}\right)^{4}} \simeq 510^{-30} h_{50}^{2}\left(\frac{\Omega_{b}}{0.05}\right)\left(\frac{f_{*}}{0.1}\right)\left(\frac{2.5}{\left(1+z_{*}\right)}\right)\left(\frac{\epsilon}{0.001}\right)\left[\mathrm{g} / \mathrm{cm}^{3}\right] .
$$

For stellar processes, $\epsilon$ is essentially determined by the IMF: $\epsilon=0.001$ for a Salpeter IMF and a low-mass cutoff $M_{\min }=0.1 \mathrm{M}_{\odot}, \epsilon=0.002$ for $M_{\text {min }}=$ $2 \mathrm{M}_{\odot}$, while it gets the usually quoted value of $\epsilon=0.007$ only for $M_{\min }>10 \mathrm{M}_{\odot}$.

Constraints from the Integrated Optical Background Let us adopt for the optical/ near-IR bolometric emission by distant galaxies between 0.1 and $7 \mu \mathrm{m}$ the value given in eq. (3). We discussed evidence that in luminous starbursts the starburst emission itself only moderately contributes to the optical spectra, the latter being largely hidden in the far-IR. Then let us assume that the optical/NIR background mostly originates in quiescent SF in spiral disks and by intermediate and low-mass stars. As observed in the solar neighborhood, a good approximation to the IMF in such relatively quiescent environments is the Salpeter law with standard low-mass cutoff, corresponding to a mass-energy conversion efficiency $\epsilon \sim 0.001$. With these parameter values, we can reproduce the whole optical background intensity of eq. (3) by transforming a fraction $f_{*} \simeq 10 \%$ of all nucleosynthetic baryons into (mostly low-mass) stars, assuming that the bulk of this process happened at $z_{*} \sim 1.5$ and $5 \%$ of the closure value in baryons (for our adopted $\mathrm{H}_{0}=50 \mathrm{~km} / \mathrm{s} / \mathrm{Mpc}$, or $\Omega_{b} h_{100}^{2}=0.012$ ):

$$
\left.\nu I(\nu)\right|_{\text {opt }} \simeq 2010^{-9} h_{50}^{2}\left(\frac{\Omega_{b}}{0.05}\right)\left(\frac{f_{*}}{0.1}\right)\left(\frac{2.5}{1+z_{*}}\right)\left(\frac{\epsilon}{0.001}\right) \mathrm{W} / \mathrm{m}^{2} / \mathrm{sr} .
$$

A local density in low-mass stars is generated in this way consistent with the observations (Ellis et al. 1996), $\rho_{b}($ stars $) \simeq 710^{10} f_{*} \Omega_{b} \simeq 3.410^{8} \mathrm{M}_{\odot} / \mathrm{Mpc}^{3}$, corresponding to a local density in metals of $\rho_{Z}$ (stars $) \simeq 710^{10} f_{*} \frac{Z}{Z} \Omega_{b} \mathrm{M}_{\odot} / \mathrm{Mpc}^{3} \simeq$ $710^{6} \mathrm{M}_{\odot} / \mathrm{Mpc}^{3}$. 
Explaining the CIRB Background Following our previous assumption that luminous starbursting galaxies emit negligible energy in the optical-UV and most of it in the far-IR, we coherently assume that the energy resident in the CIRB background (see eq.[2]) originates in star-forming galaxies at median $z_{*} \simeq 1.5$. The fraction of baryons processed during this phase and the conversion efficiency $\epsilon$ have to explain the huge energy content of the CIRB without contributing appreciably to the number of stellar remnants locally observed. The only plausible solution would then be to change the assumptions about the stellar IMF characterizing the starburst phase, for example to a Salpeter distribution cutoff below $M_{\text {min }}=2 \mathrm{M}_{\odot}$, with a correspondingly higher efficiency $\epsilon=0.002$. This may explain the energy density in the CIRB (eq. [2]):

$$
\left.\nu I(\nu)\right|_{F I R} \simeq 4010^{-9} h_{50}^{2}\left(\frac{\Omega_{b}}{0.05}\right)\left(\frac{f_{*}}{0.1}\right)\left(\frac{2.5}{1+z_{*}}\right)\left(\frac{\epsilon}{0.002}\right) \mathrm{W} / \mathrm{m}^{2} / \mathrm{sr},
$$

assuming that a similar fraction of baryons, $f_{*} \simeq 10 \%$, as processed with low efficiency during the "inactive" secular evolution, is processed with higher efficiency during the starbursting phases, producing a two times larger amount of metals: $\rho$ (metals) $\sim 1.410^{7} \mathrm{M}_{\odot} / \mathrm{Mpc}^{3}$. Note that by decreasing $M_{\min }$ during the SB phase would decrease the efficiency $\epsilon$ and increase the fraction of processed baryons $f_{*}$, and would begin to exceed the locally observed mass in stellar remnants. This scheme is made intentionally extreme, to illustrate the point. The real IMFs are likely to be more complex, e.g., including a low-mass flattening.

A direct prediction of this scheme is that most of the metals produced during the starburst phase have to be removed by the galaxies to avoid exceeding the locally observed metals in galactic stars and that substantial amounts of metals originating in the $S F$ processes producing the CIRB are hidden in cosmic media. The metals observed in the X-ray emitting plasmas of clusters and groups of galaxies (considered as closed boxes from a chemical point of view, as well as representative samples of the universe) provide interesting support to this prediction.

\subsection{A Two-Phase Star-Formation: Origin of Galactic Disks and Spheroids}

Our best-fit model for IR galaxy evolution implies that star formation in galaxies has proceeded along two phases: a quiescent one taking place during most of the Hubble time, slowly building stars with a standard IMF from the regular flow of gas in rotationally supported disks; and a transient active starbursting phase, recurrently triggered by galaxy mergers and interactions. During the merger, violent relaxation redistributes old stars, producing de Vaucouleur profiles typical of galaxy spheroids, while young stars are generated following a top-heavy IMF.

Because of the geometric (thin disk) configuration of the diffuse ISM and the modest incidence of dusty molecular clouds, the quiescent phase is only moderately affected by dust extinction, and naturally produces most of the optical/NIR background (including NIR emission by early-type galaxies completely deprived of an ISM). 
The merger-triggered active starburst phase is characterized by a large-scale redistribution of the dusty ISM, with bar-modes and shocks compressing a large fraction of the gas into the inner galactic regions and triggering formation of molecular clouds. As a consequence, this phase is expected to be heavily extinguished and naturally produces the CIRB. Based on dynamical considerations, we expect that during this violent $S B$ phase the elliptical and $S 0$ galaxies are formed in the most luminous IR SBs at higher-z (corresponding to the SCUBA source population), whereas galactic bulges in later-type galaxies likely originate in lower IR luminosity, lower-z SBs (the ISO mid-IR population).

\section{Conclusions}

Studies of galaxy formation and evolution at long wavelengths benefit from the unique advantage of combining deep extragalactic imaging surveys with knowledge of the integrated emission at any redshift provided by the cosmic IR background. The pioneering explorations of the distant universe at IR wavelengths we have summarized in the previous sections already give vivid illustrations of the new perspectives achievable through these new cosmological channels. These results challenge those obtained from optical-UV observations, by revealing luminous and very luminous phases in galaxy evolution at substantial redshifts, likely corresponding to violent events of star-formation in massive systems. This is bringing significant refinements to present schemes of galaxy formation.

\section{References}

Aussel, H., 1998, PhD Thesis, CEA Saclay, Paris

Aussel, H., Cesarsky, C., Elbaz, D., \& Starck, J. L. 1999, A\&A, 342, 313

Barger, A., Cowie, L., Mushotzky, R., \& Richards, E. 2000, ApJ, submitted (astro-ph/0007175)

Burigana, C., Danese, L., De Zotti, G., Franceschini, A., et al. 1997, MNRAS, 287, L17

Bertoldi, et al. 2000, Highlights of Astronomy 12, in press (astroph/0010553)

Cohen, J., et al. 1999, in press (astro-ph/9912048)

Coppi, P., \& Aharonian, F. 1999, Astropart. Phys., 11, 35

Dole, H., Gispert, R., Lagache, G., et al. 2000, in ISO Beyond Point Sources, Studies of Extended Infrared Emission, ed. R. Laureijs, K. Leech \& M. Kessler, ESA-SP 455, 167

Elbaz, D., et al. 1999, A\&A, 351, L37

Elbaz, D., et al. 2000, in preparation

Ellis, R. S., Colless, M., Broadhurst, T., Heyl, J., \& Glazebrook, K. 1996, MNRAS, 280, 235

Fabian, A., et al. 2000, MNRAS, 315, 8

Finkbeiner, D. P., Davis, M., \& Schlegel, D. J. 2000, ApJ, 544, 81

Fixsen, D. J., et al. 1998, ApJ, 508, 123

Flores, H., Hammer, F., Thuan, T., et al. 1999, ApJ, 517, 148 
Franceschini, A., Mazzei, P., De Zotti, G., \& Danese, L. 1994, ApJ, 427, 140

Franceschini, A., et al. 2000, in preparation

Franceschini, A. 2000, in Galaxies at High Redshifts, ed. F. Sanchez, I. Perez-

Fournon, M. Balcells, \& F. Moreno-Insertis (Cambridge: Cambridge University Press), (astroph/0009121)

Gispert, R., Lagache, G., \& Puget, J.-L. 2000, A\&A, 360, 1

Gorjian, V., Wright, E. L., \& Chary, R. R. 2000, ApJ, 536, 550

Harwit, M. 1999, ApJ, 510, L83

Harwit, M., Protheroe, R. J., \& Biermann, P. L. 1999, ApJ, 524, L91

Hauser, M. G., Arendt, R. G., Kelsall, T., et al. 1998, ApJ, 508, 25

Hornschemeier, A. E., Brandt, W. N., \& Garmire, G. P. 2000, ApJ, in press (astro-ph/0004260)

Kormendy, J., \& Sanders, D. 1992, ApJ, 390, 53

Krawczynski, H., Coppi, P. S., Maccarone, T., \& Aharonian, F. A. 2000, A\&A, 353,97

Lagache, G., Abergel, A., Boulanger, F., Desert, F.X., \& Puget J.-L. 1999, A\&A, 344,322

Lilly, S. J., et al. 1999, ApJ, 518, 641

Madau, P., \& Pozzetti, L. 2000, MNRAS, 312, L9

Mushotzky, R. F., \& Loewenstein, M. 1997, ApJ, 481, L63

Oliver, S., et al. 2000, MNRAS, 316, 749

Poggianti, B. M., Bressan, A., \& Franceschini, A. 2000, ApJ, in press (astroph/0011160)

Puget J.-L., et al. 1996, A\&A, 308, L5

Puget, J.-.L., Lagache, G., Clements, D. L., et al. 1999, A\& A, 345, 29

Rigopoulou, D., Franceschini, A., Aussel, H., et al. 2000, ApJ, 537, L85

Risaliti, G., Gilli, R., Maiolino, R., \& Salvati, M. 2000, A\& A, 357, 13

Roche, N., \& Eales, S. A. 1999, MNRAS, 307, 703

Rowan-Robinson, M., et al. 1997, MNRAS, 289, 482

Stanev, T., \& Franceschini, A. 1998, ApJ, 494, L159

Stecker, F., De Jager, O., \& Salamon, M. 1992, ApJ, 390, L49

Vigroux, L., et al. 1998, in The Universe as seen by ISO, ESA-SP 427, 805

Xu, C. 2000, ApJ, 541, 134

\section{Discussion}

Tom Shanks: Several of your VLT IR spectra of ISO sources appear to show broad lines as if they were QSOs. Can you comment?

Alberto Franceschini: Our VLT observations of the $\mathrm{H}_{\alpha} / \mathrm{NII}$ lines for high$z$ ISO sources have been done in the low resolution mode. Our preliminary attempts to recognize broadened components has not yet provided conclusive results. Only in one case did we find an inverted value for the $\mathrm{H}_{\alpha} / \mathrm{NII}$ flux ratio, 
indication of an AGN. We are presently working on higher-resolution observations which should eventually provide better constraints. 